\title{
Interdiscursivité et transmissions orales dans le domaine de l'aide à la personne avec les personnes âgées
}

\section{Interdiscursivity and oral transmissions in the context of caregiving with seniors}

\author{
Marie Lefelle ${ }^{1}$ \\ ${ }^{1}$ Laboratoire Grammatica, Université d'Artois, France
}

\begin{abstract}
Résumé. Les professionnels qui s'occupent des personnes âgées dans les établissements de santé possèdent plusieurs identités, à la fois aidants dans la relation avec la personne âgée et spécialistes de la pratique dans les interactions interprofessionnelles, ils s'adaptent en permanence au contexte, aux individus et à la réalité qui s'imposent à eux. Cette double identité pose des questions quant aux compétences de ces professionnels qui doivent être capables de présenter des qualités humaines d'écoute, d'empathie dans leurs échanges avec les personnes âgées mais également des capacités strictement professionnelles englobant la maîtrise du lexique spécialisé et les implicites spécifiques à la pratique dans les communications interprofessionnelles. Pour cet article, nous nous servirons d'un corpus récolté dans un organisme de formation des professionnels du soin et de l'aide et dans un établissement de santé pour les personnes âgées. Nous nous intéresserons aux communications interprofessionnelles à travers la problématique de l'interdiscursivité dans les transmissions orales. Les professionnels se servent effectivement de discours pour justifier des soins apportés ou pour rapporter des éléments qu'ils jugent importants de soumettre aux autres professionnels dans le cadre du suivi des soins pour la personne âgée. Nous verrons que les discours partagés en transmissions orales relèvent d'implicites spécifiques à la pratique professionnelle maîtrisés par tous les membres de la communauté de pratique.
\end{abstract}

\begin{abstract}
Professionals who take care of the elderly, particularly in health establishments, have several identities, both helpers in the relationship with the elderly and specialists in practice in interprofessional interactions, they constantly adapt to the context, to the individuals and the reality. This double identity raises questions about the skills of these professionals who both must be able to present human qualities of listening, empathy in their exchanges with the elderly but also strictly professional capacities including mastery of the specialized lexicon and the practice specific implicit in interprofessional communications. For this article, we will use a corpus collected from both a training organization for care and aid professionals and a health facility for the elderly. We will focus on interprofessional communications through the issue of interdiscursiveness in oral transmissions. Professionals do use discourse to justify the care provided or to report elements that they consider important to submit to other professionals as part of the follow-up of care for the elderly. Through this issue of interdiscusivity, we will see that the discourses shared in oral transmissions are implicit specific to professional practice, mastered by all members of the community of practice.
\end{abstract}


Les professionnels du soin et de l'aide utilisent les transmissions orales pour communiquer au sein de leur communauté de pratique. Cet exercice quotidien fait partie intégrante de la pratique et participe aux soins de la personne âgée. Dans une logique de réflexivité collective et s'agissant d'un exercice oral effectué quotidiennement, les professionnels utilisent ce que qu'ils ont vu et entendu pour transmettre les informations pertinentes à leurs pairs ainsi qu'au personnel médical. Le présent article s'attardera donc sur la problématique de l'interdiscursivité au sein des transmissions orales. La notion de dialogisme a déjà été utilisée pour l'analyse des discours dans la sphère de l'enseignement, elle nous semble tout aussi pertinente pour comprendre la prise de parole des professionnels du soin et de l'aide au sein de l'exercice particulier que constituent les transmissions orales. À travers l'étude et l'analyse des trois types de dialogisme (interdiscursif, intralocutif et interlocutif) nous tenterons donc de comprendre la logique et la réflexivité des professionnels du soin et de l'aide dans l'utilisation des discours lors des transmissions orales.

\title{
1 L'interdiscursivité et le dialogisme
}

La langue que nous parlons est arbitraire, elle suppose une vision du monde qui nous précède à travers une histoire particulière, une géographie, des individus qui l'ont influencée. Une culture donnée modifie le rapport au réel, c'est ce que sous-entend Sapir dans son hypothèse : « Le langage est un moyen de communication purement humain et non instinctif, pour les idées, les émotions et les désirs, par l'intermédiaire d'un système de symboles créés à cet effet. » (2001 : 15). Or ces symboles ont été conçus arbitrairement par une collectivité particulière qui possède son propre cadre, sa propre catégorisation du monde :

\begin{abstract}
«Parler suppose l'usage d'une langue dite naturelle. Les interactions verbales sont construites dans des langues particulières qui imposent certaines contraintes aux interactants et à leur travail d'interprétation et de production. Elles imposent leurs organisations syntaxiques, prosodiques ou lexicales spécifiques.

De ce point de vue, on peut considérer que les langues particulières fournissent aux interactants un cadre tout fait, arbitraire et déjà là de catégorisation du monde. Mais cette catégorisation linguistique a aussi ses limites ou ses failles, et en particulier des limites lexicales. Les langues ne fournissent pas on le sait, de dénominations précises et satisfaisantes pour exprimer toutes les situations sociales complexes, ni pour toutes les expériences spécifiques de chaque sujet. Avec le matériau sémiotique des langues, nécessairement collectif, chacun doit se « débrouiller » pour exprimer le singulier de son expérience, de ses désirs et de ses savoirs ». (Boutet, 1995 : 256).
\end{abstract}

Si la langue conditionne la vision du monde à travers son prisme, les discours qui ont précédé l'influencent tout autant. À ce sujet Charaudeau et Maingueneau affirment que « tout discours est traversé par l'interdiscursivité, il a pour propriété constitutive d'être en relation multiforme avec d'autres discours, d'entrer dans l'interdiscours. » (2002:324)

Nous pourrions dire que la langue fournit déjà un cadre contraint mais que les discours qui ont précédé, contraignent et rendent encore moins possible l'improvisation du locuteur. C'est par ailleurs d'autant plus vrai dans un milieu professionnel où le dire produit un rendement. Si la première fonction d'un professionnel est d'aider, c'est que cette aide (qu'elle soit verbale ou dans les actes) produit une forme de productivité rémunérable. Bien qu'elle soit 
plus visible lorsqu'elle laisse des traces dans le réel, cette aide se réalise aussi dans le langage (de manière indirecte également dans les transmissions orales). Pour comprendre comment les discours influencent la pratique professionnelle nous nous intéressons à la problématique de l'interdiscursivité à travers le dialogisme :

« Le dialogisme consiste donc en l'orientation de tout discours (quel que soit son format: tour de parole, article de presse, discours politique, article scientifique, texte littéraire, etc.) vers d'autres discours, sous forme de dialogue interne avec ceux-ci, et ce triplement :

- vers des discours réalisés antérieurement par des tiers, le plus souvent sur le même objet ;

- vers le tour de parole antérieur de l'allocutaire dans les genres dialogaux et, tant dans le dialogal que dans le monologal, vers la réponse de l'allocutaire qu'il sollicite et sur laquelle il anticipe ;

- vers lui-même, le locuteur étant son premier allocutaire "». (Bres, $2017: 3$ ).

Pour comprendre comment les discours influencent la pratique, nous étudierons leurs influences au sein de la pratique professionnelle en particulier lors des transmissions orales. Cette étude de l'influence des discours a déjà été réalisée pour le domaine de l'enseignement, elle a ainsi permis de mettre en lumière l'utilisation des discours au sein des cours magistraux :

La notion de dialogisme a toute sa pertinence pour l'analyse des discours de la sphère d'activité de l'enseignement, tant ceux des enseignants que ceux des élèves et étudiants.

En tant que reposant sur la transmission et l'appropriation de connaissances, le discours enseignant passe par la reformulation interdiscursive des discours de la discipline ; c'est notamment le cas du cours magistral : reformulations explicites, implicites, allusives, digression sont analysées dans les articles de Parpette \& Bouchet (cours magistral en FLE), et de Raviez \& Mangiante (cours magistral de littérature), les auteurs s'attachant à décrire comment le dialogisme interdiscursif se combine avec le dialogisme interlocutif : l'enseignant façonne son discours en fonction des connaissances qu'il prête à ses étudiants, et en anticipation constante sur les difficultés de compréhension qu'il imagine qu'ils peuvent rencontrer. Plus spécifiquement, parce que l'acquisition du savoir est cumulative, l'enseignant revient sur ce qui a été dit antérieurement, c'est-à-dire interagit avec ce que Parpette et Bouchet proposent de nommer pertinemment intradiscours, pour désigner les données évoquées précédemment dans son discours, cette interaction pouvant prendre des formes explicites dans le cours magistral (« petit rappel sur ce qu'on a fait la semaine dernière »), ou fortement implicites dans les séquences scolaires en milieu gitan analysées par Auger et Dufour, lorsque les répétitions d'un mot ou les questions de l'enseignante convoquent un discours antérieur 
tenu en classe : ce qui a été « vu », ce que l'élève « devrait

savoir ». (Bres, $2017: 7)$.

Dans la pratique professionnelle à proprement parler, nous supposons donc que l'utilisation des discours est corrélée avec le milieu professionnel étudié puisque soumise aux contraintes, à la logique de soin, de rendement ainsi qu'aux impératifs du secteur.

\section{Le domaine de l'aide à la personne avec les personnes âgées}

Le domaine de l'aide à la personne est un secteur en pleine expansion en France et dans le reste du monde du fait notamment du vieillissement de la population. Les besoins en personnels dans ce domaine y compris le recrutement de personnels étrangers pour couvrir ces besoins ne feront que progresser dans les décennies suivantes en raison du vieillissement de la population et en particulier auprès des personnes dites dépendantes (handicapées, polyhandicapées ou vieillissantes) :

«Au 1er janvier 2012, en France métropolitaine, 1,17 million de personnes âgées étaient dépendantes [...] A l'horizon 2060, selon le scénario intermédiaire des projections de dépendance, le nombre de personnes âgées dépendantes atteindrait 2,3 millions ». (Trivalle, $2016: 6$ ).

\subsection{La part langagière dans le domaine de l'aide à la personne avec les personnes âgées}

La part langagière en particulier dans les métiers de services occupe une part essentielle de la pratique professionnelle, elle était pourtant autrefois synonyme d'oisiveté :

« Le taylorisme avait développé une conception de l'activité de langage au travail selon laquelle parler et travailler sont des activités antagonistes : parler est un bavardage inutile qui fait perdre les précieuses secondes requises pour l'effectuation de la procédure opératoire et qui bloque l'automatisation du geste ». (Boutet et Gardin, 2005 : 101).

Ce n'est bien entendu plus le cas, en particulier pour les métiers de services où la rentabilité et l'efficacité du professionnel passent par l'action mais aussi par la parole :

« La part du contrôle des dispositifs techniques au moyen des activités sémiotiques (activités d'écriture et de lecture, sur écrans ou sur papier), la part des activités de délibération, de coordination (réunions, communications téléphoniques), la part de toutes ces " techniques intellectuelles " s'accroissent aux dépens des activités physiques de transformation directe de la matière. Le langage est désormais reconnu comme un facteur de productivité (Zarifian, 1996); il ne s'oppose plus au rendement économique, mais, au contraire, le favorise. La mobilisation de l'intelligence et des savoirs des salariés, l'exigence de coopération passent par une prise en compte de l'activité langagière des agents. La communication, l'accès à l'information deviennent centrales dans ces formes d'organisation du travail ; savoir communiquer devient une compétence professionnelle ». (Boutet et Gardin, 2005). 
Dans le domaine de l'aide à la personne en particulier, la part langagière est au moins aussi importante que les actes du professionnel.

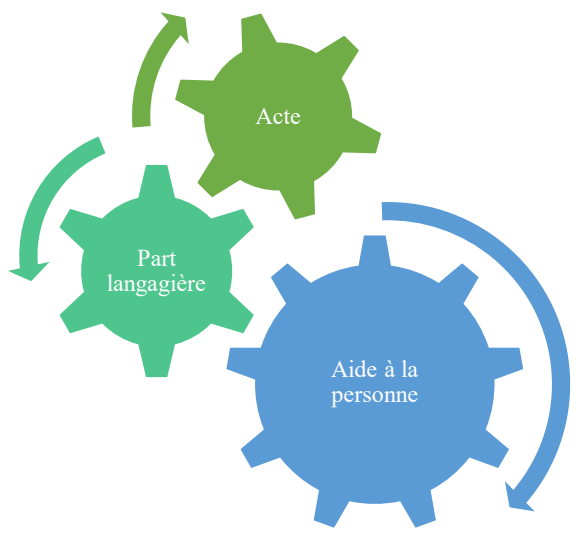

Figure 1. Aide à la personne.

L'aide n'est pas à entendre comme seulement présente dans les actes d'un professionnel. En effet, une aide peut aussi appartenir exclusivement au domaine du langagier, c'est par exemple le cas de professionnels qui rassurent une personne âgée fragilisée. L'aide à la personne englobe donc une aide qui ferait référence aux actes du professionnel mais aussi à son langage. Il existe des situations où l'aide ne correspond qu'à des actes mais il existe aussi des situations où l'aide ne correspond qu'au domaine du langage (bien qu'il s'agisse le plus souvent d'un mélange des deux). Par ailleurs la part langagière des professionnels dans le domaine de l'aide à la personne n'est pas uniquement constituée de communications avec l'aidé. Les professionnels du domaine sont ainsi amenés à interagir avec la communauté de pratique dont font partie les professionnels de santé (infirmière, médecin etc.) lors des transmissions orales par exemple. Dans une certaine mesure, travailler dans une communauté de pratique incluant des professionnels de santé, oblige les professionnels s'occupant des personnes âgées, à comprendre et à utiliser un vocabulaire médical lors des interactions entre professionnels. En effet, la relation d'aide suppose que les professionnels ont compris les paramètres de cette aide et concernant des personnes âgées, cela inclut une connaissance des pathologies, des mesures prises sur le plan médical et des contre-indications que cela peut entraîner par exemple au niveau d'un soin non médical.

\subsection{Les professionnels du soin et de l'aide}

Un professionnel de l'aide à la personne n'est pas un professionnel de santé, il est surtout chargé d'aider la personne dans les gestes du quotidien y compris lorsqu'elle ne peut plus verbaliser ses besoins. Il réalise des soins non médicaux tels que le change de la protection, la toilette et la réfection du lit par exemple. Au sujet des employés au domicile de particuliers, Mourlhon-Dallies disait ceci :

«Mais en quoi consistent-ils exactement? L'indéfinition relative de ces emplois nous paraît tenir à leur oscillation entre l'aide et le soin. Le soin s'entend ici à trois niveaux :

- $\quad$ l'entretien du domicile (prendre soin de)

- le fait de surveiller l'état de santé de personnes dont on a la charge (enfants en bas âge, qu'il faut souvent soigner, 
personnes âgées que l'on guide dans l'observance des traitements prescrits)

- mais aussi soins non médicamenteux que l'on prodigue, ou " menus soins ", qui renvoient plutôt à la maîtrise de l'hygiène quotidienne et sont censés donner à leur bénéficiaire une allure " soignée ». Les soins en question n'ayant pas un caractère strictement médical, on bascule alors dans la catégorie de l'aide apportée à des catégories de personnes non autonomes (enfants, personnes très âgées, malades, handicapés, personnes en convalescence) ». (2011: 76)

On se situe entre le soin et l'aide, c'est pourquoi au sein du présent article, nous utilisons le terme générique de professionnel de l'aide et du soin pour désigner ces professionnels. En outre, nous utilisons cette dénomination car le statut des salariés et le type de diplôme obtenu ne déterminent pas le type de tâches qu'auront à réaliser les professionnels. On assiste ainsi à une confusion des statuts et à des responsabilités interchangeables, c'est par exemple le cas des aides médico-psychologiques et des aides-soignantes qui en définitive se chargent des mêmes soins concernant les personnes âgées.

\subsection{Changement de posture}

Les différents types d'interactions qui ont été récoltées pour le corpus ont permis de mettre en avant le changement de posture du professionnel du soin et de l'aide qui adapte son discours en fonction du locuteur en présence. Le futur professionnel apprendra et intégrera non seulement les codes et les rites de son environnement de travail mais il modifiera son langage au regard de la situation et de l'interlocuteur. En exemple cet extrait issu de la formation des professionnels de l'aide et du $\operatorname{soin}^{1}$ sur la réfection du lit inoccupé :

(1) Professeure : Première chose quand on rentre dans la chambre du patient qu'est-ce qu'on dit? Mandy vas-y t'es là

Élève 4 : Bonjour Monsieur!

Professeure : $\mathrm{Hm} \mathrm{hm}$

Élève 4 : Euh

Professeure : Donc Madame Luc vous l'appelez Madame

Élève 4: On va vous faire euh la réfection du lit

Professeure: On va changer vos draps, réfection du lit, la personne euh elle sait pas trop ce que c'est ça, vous qui avez en tête cette technique là mais la personne âgée. Bah monsieur voilà fin...Madame on va dire dans les situations, vos draps ils sont souillés on va les changer, on va changer vos draps, c'est plus simple que dire on va faire la réfection du lit, même si dans ta tête c'est ça que tu as.

$\mathrm{Au}$ sein de la formation ASSP, les élèves apprennent à moduler leur discours en fonction de l'interlocuteur, réfection de lit pour le professionnel dans sa pratique, changer les draps, expression issue de la langue générale, pour interagir avec la personne âgée. On observera ainsi l'apparition de lexique spécialisé, c'est-à-dire d'un lexique spécifique au professionnel qui s'emploie dans un cadre où il est en interaction avec un autre professionnel et/ou en réflexion personnelle, ce lexique et la pratique associée à celui-ci demeurent souvent étrangers à une personne ne faisant pas partie de la communauté de pratique.

\footnotetext{
${ }^{1}$ Langage parlé issu de la formation des professionnels de l'aide et du soin.
} 
Le cadre énonciatif, c'est-à-dire les protagonistes du discours (émetteur et destinataire) et la situation de communication (les circonstances spatio-temporelles et les conditions générales de la production/réception du message) influencent donc les interactions.

Les discours des professionnels sont en effet toujours influencés par un contexte et un cotexte. Le type de public auquel est confronté le professionnel, va modifier le contenu de son discours, le contenu d'une interaction prise dans une relation d'aide entre une personne âgée et un professionnel, va grandement différer d'une conversation interprofessionnelle. Par ailleurs, le discours du professionnel est aussi influencé par tous les discours qu'ils l'ont précédés, c'est ce que nous verrons en abordant l'interdiscursivité et le dialogisme.

Le contexte a une grande influence sur le contenu du discours et la posture du professionnel, c'est une caractéristique que l'on pourrait par ailleurs attribuer aux services de soin mais aussi de santé comme l'atteste cet extrait issu du livre de Grosjean et Lacoste sur la communication et l'intelligence collective à l'hôpital :

«Le travail des soignants autres que les médecins demeure moins connu. Une étude de la coopération entre sage-femme et parturiente durant l'accouchement (Grosjean, 1993) a cependant permis de montrer le rôle structurant de la voix dans le travail de la sage-femme : celle-ci en utilise les modulations pour créer des « contextes» ou « cadres » qui vont guider l'accouchement; elle dispose de plusieurs voix, dont elle joue selon les circonstances : voix « empathique », qui réconforte, voix « professionnelle », qui instaure un nécessaire rapport d'autorité. La modulation de la voix est un fait bien connu des malades hospitalisés : la voix haut perché avec laquelle l'infirmière entre dans une chambre en mimant l'enthousiasme (« Alors on est en forme aujourd'hui ! »), ou la voix protectrice qui enjoint au malade d' « être bien raisonnable " sont presque devenues des stéréotypes. Changement de voix et changement de cadre participatif vont de pair : en passant d'un malade grave qu'on veut réconforter à une interaction avec une jeune collègue, qu'il faut par exemple rappeler à l'ordre, puis à une question adressée à un médecin, l'infirmière change chaque fois de partenaire, de mode d'engagement, de type d'action sociale. Le passage rapide d'une position à l'autre est caractéristique de certaines professions au contact avec le public. (Grosjean et Lacoste, 1999 : 26).

Si l'on veut étudier les domaines de la santé et du soin, on peut ignorer le contexte au risque d'étudier la langue de manière superficielle et irréaliste. Si le professionnel module sa posture dans sa relation avec le patient, c'est d'autant plus vrai lorsque la catégorie de public avec lequel il interagit se modifie en passant du patient au pair comme dans les transmissions orales. 


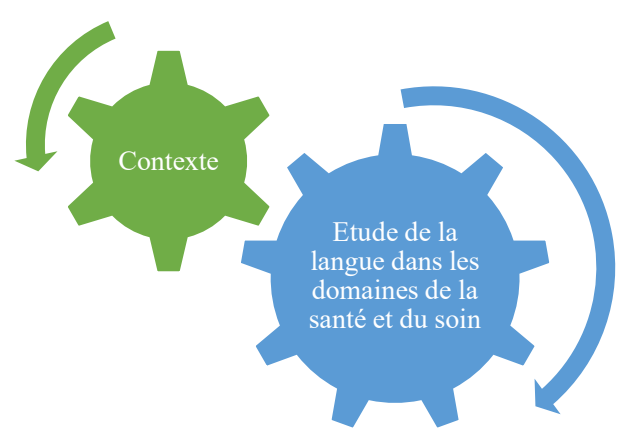

Figure 2. Contexte et étude de la langue dans les domaines de la santé et du soin.

\section{Corpus et méthodologie}

La recherche en contexte réel a permis de collecter un large corpus (vidéo et audio) issu d'un établissement de formation (le lycée Joliot-Curie à Oignies et la filière $\mathrm{ASSP}^{2}$ ) et d'un EHPAD $^{3}$ (Stéphane Kubiak du groupe la Vie active qui possède 14 établissements de ce type en France).

Ce corpus est constitué d'interactions entre professeures et futurs professionnels de l'aide et $\mathrm{du}$ soin, d'interactions aidant-aidé ainsi que d'interactions interprofessionnelles, il est composé de langage parlé. Ce langage en particulier est porteur des marques du réel, d'interruption du discours, de changement de sujet, de répétition, de contraction, il demeure ainsi parfois difficile à comprendre et à interpréter notamment dans l'utilisation des discours fait par les professionnels, c'est par ailleurs d'autant plus vrai lorsque l'on ne fait pas partie de la communauté de pratiques. Ce sont donc les interactions interprofessionnelles auxquelles nous nous intéresserons dans le cadre du présent article. Comme nous avons pu l'indiquer, elles se distinguent par une modification du lexique employé mais également par une logique et une réflexivité spécifique au contexte et aux interlocuteurs en présence. Nous avons ainsi relevé pour l'ensemble du corpus, les discours qui influencent la pratique en tentant parfois de comprendre ce qui motivait les professionnels dans leurs utilisations. Bien que pour le présent article le corpus collecté en formation ne soit pas utilisé (puisque nous nous intéresserons strictement aux transmissions orales), il est à signaler que la formation a également été analysée dans son utilisation des discours. Nous avons ainsi pu nous rendre compte qu'une partie des discours transmis en formation étaient effectivement réutilisés en contexte professionnel :

Extrait du corpus $\mathrm{n}^{\circ} 8$ issu de la formation

(2) Élève: Vous voulez que je lui dise quoi? Professeure : Bah je ne sais pas 'y a un grand soleil ce matin j'sais pas moi vous avez bien dormi?

Extrait du corpus $n^{\circ} 8$ issu de la formation

\section{Extrait du corpus $n^{\circ} \mathbf{3 1}$ issu de l’EHPAD}

\footnotetext{
${ }^{2}$ Accompagnement, Soins et Services à la Personne.

${ }^{3}$ Établissement d'Hébergement pour Personnes Âgées Dépendantes.
} 
(3) Professionnelle 2 : Vous avez bien dormi?

Résidente : Ça va, ça va

Professionnelle 2 : ouais

Résidente : J'ai pris mes médicaments mais pfou!

Professionnelle 2: Ouais j'me doute hein, mais bon on fait au mieux à chaque fois, $j$ 'vais monter un p'tit peu le lit

Résidente : D'accord

Professionnelle 1 : Vous avez vu il fait du beau soleil aujourd'hui

Résidente : Oui

Professionnelle 1 : Ça annonce une belle journée!

Résidente : Ouais

\section{Dialogisme}

Dans le domaine de l'aide à la personne, l'aide se partage donc entre une aide verbale et une aide dans les actes. Si la première fonction d'un professionnel est d'aider c'est que celle-ci (qu'elle soit verbale ou dans les actes) produit une forme de productivité rémunérable or un professionnel de l'aide et du soin est aussi amené à transmettre les discours dans le cadre d'interactions interprofessionnelles. Ce sont en effet ces dernières qui assurent la transmission des informations qui permettent d'adapter et d'ajuster les soins pour la personne âgée. Les professionnels de l'aide et du soin transmettent des discours à la communauté de pratique professionnelle. Pour comprendre comment les discours influencent la pratique professionnelle, nous nous intéressons à la problématique de l'interdiscursivité à travers les trois types de dialogisme : interdiscursif, intralocutif et interlocutif. Les transmissions orales constituent donc une forme de réunion professionnelle spécifique au milieu de la santé et du soin, c'est lors de ces réunions que les professionnels se rassemblent pour discuter des résidents et des informations pertinentes à partager au groupe et pour cela ils réinvestissent des discours entendus ou issus du dit intérieur.

\subsection{Dialogisme interdiscursif}

L'apparition de dialogisme interdiscursif, c'est-à-dire de discours antérieurs rapportés, s'opère au sein des transmissions orales. Au cours de ces transmissions, le professionnel transmet les communications qu'il a eues avec le résident ainsi que celles qu'il a eues avec ses collègues ou encore les informations qu'il a reçues et qu'il considère pertinent de partager avec ses pairs.

Le professionnel au cours des transmissions, considère qu'un changement de comportement du résident est pertinent à partager, c'est par exemple le cas d'une interaction impliquant de l'agressivité exprimée par le résident :

(4) Professionnelle 5 : Alors Julia très énervée mais vraiment... elle est un coup elle m'a dit euh heureusement que tu es pas à la salle depuis ce matin parce que ta voix aigüe là ah mon dieu nan mais vraiment en fait elle est pas contente de tout. Euh Janine elle lui propose un café elle s'est mise à l'insulter comme je ne sais quoi euh pour un rien elle s'énerve pour un rien vraiment elle hurle depuis ce matin elle hurle.4

Professionnelle 1 : Elle a pas eu d'appel de mauvaise nouvelle des choses comme ça?

\footnotetext{
${ }^{4}$ Langage parlé issu des transmissions orales.
} 
Professionnelle 5 : Nan, nan, nan mais elle nous insulte et tout hein voilà. Après elle nous dit parce ce matin quand j'ai fait sa toilette quand j'suis partie faire son dos et tout euh Émilie elle est venue dans la chambre elle était nue donc ça l'a énervée mais j'lui ai dit j'fais ben ah si c'est grave mais elle a pas fait exprès fin elle est venue te chercher fin quoi Émilie j'sais pas si c'est vraiment ça qui l'a énervée

Malheureusement dans un contexte de soin et de dépendances souvent fortes, l'agressivité est un phénomène fréquent dans la relation avec la personne âgée, c'est d'autant plus vrai puisqu'une proportion significative de personnes âgées souffre de la maladie d'Alzheimer avec une perte de repères pouvant entraîner de très diverses réactions : de la confusion, de la tristesse par exemple mais également de l'agressivité. Les professionnels se transmettent ce genre de discours puisque l'agressivité de la personne âgée a un impact sur les soins. Prévenir ses pairs et la communauté médicale se révèlent dès lors essentiels à la prise en charge (d'autant plus si ce type de comportement est récurrent). Le professionnel considère également de partager au groupe un changement de comportement tel qu'un refus, comme dans cet extrait où le professionnel rapporte un discours où la résidente refuse de mettre ses nouvelles chaussures :

(5) Professionnelle 3 : Et après j'ai Laura qui a pas voulu mettre ses nouvelles chaussures

Professionnel non identifié : Elle nous fait souvent ça vu qu'elle a mal hein ouais elle dit que ça lui fait mal

Professionnelle 3 : Nan moi elle m'a dit j`suis tombé dimanche du coup je les mets pas

Professionnel non identifié : Et qui?

Professionnelle 3 : Laura

Le refus est également relativement fréquent dans la prise en charge, il impacte tout autant les soins que l'agressivité. Dans un contexte d'aide, il alourdit la prise en charge en temps et en ressources pour les professionnels (temps réservé à la négociation, moins de personnes âgées prises en charge, etc.)

Un changement positif dans le comportement de la personne âgée est également signalé :

(6) Euh Monsieur A euh au top de la forme ce matin franchement euh (en riant) il était nickel quoi j'l'ai jamais vu comme ça il faisait des (bruits avec la bouche imitant un bisou) j'dis bon d'accord nickel

Dans cet extrait, la professionnelle souligne ainsi la particulière bonne humeur du résident. En effet, une amélioration de l'état psychique de la personne âgée indique une prise en charge plus aisée pour le professionnel, ces discours sont dont jugés importants à transmettre lors des transmissions.

De plus le professionnel considère également pertinent de partager les discours qu'il a eus avec le résident ou d'autres professionnels et qui indiquent l'apparition de pathologie, d'une dégradation de l'état de santé du résident comme c'est le cas ici pour l'apparition de glaires de sang dans les selles :

(7) Professionnelle 2 : Madame D euh c'est euh... Martine qui a fait sa toilette ce matin et elle dit que elle avait eu des selles, beaucoup de selles et elle dit que dans ses selles elle a trouvé euh quelque chose qui ressemblerait à un glaire mais de sang, avec du sang

Professionnelle 1 : D'accord

Ou encore de douleurs exprimées par le résident : 
(8) Professionnelle 2: Bah j'sais qu'elle se plaint souvent de son ventre après elle est focalisée là-dessus euh mais là pour le coup si y a des selles liquides euh

L'apparition de pathologie ou de douleur a également un impact sur les soins donnés à la personne âgée, ils peuvent également, dans un contexte de complémentarités des capacités professionnelles, entraîner une prise en charge supplémentaire comme la nécessité de faire appel à un professionnel de santé.

Le professionnel considère qu'il est pertinent de partager au groupe, une interaction avec une résidente qui exprime sa volonté dans la demande par exemple d'une chaise percée :

(9) Professionnelle 1 : je sais mais la chaise percée elle avait été retirée dans le cadre où elle a eu sa fracture du col du fémur et du coup ça l'a favorisée à se lever de son lit pour aller sur la chaise percée donc du coup on avait mis ça on avait retiré la chaise percée sauf que maintenant elle est plein de fois elle sort de son lit elle va dans la salle de bain donc du coup on s'est dit c'est un peu bête donc on lui a demandé hier en PPA. Est-ce qu'elle souhaitait qu'on remette en place une chaise percée? Elle a dit que ça l'aiderait beaucoup parce qu'elle urine beaucoup comme elle est sous diurétique

Ou encore ici d'un résident qui souhaiterait avoir son médicament plus tôt dans la soirée :

(10) Professionnel non identifié : Monsieur L demande de nuit si on peut lui donner avant

Nous le constatons, toutes les demandes faites par un résident qui entrainent une modification des soins est transmis à la communauté de pratique qui se justifie en faisant appel aux discours transmis. En outre une dégradation d'un état général peut être signifié par le résident lui-même :

(11) Professionnelle 8: Il a pas été exigeant rien du tout euh il regrette d'être allé à l'hôpital parce que il dit que bin en gros ça l'a affaibli davantage et qu'il peut plus rien faire du tout

Dans cet extrait, le professionnel partage le discours d'un résident qui exprime le regret d'être allé à l'hôpital en ajoutant qu'il se sentait affaibli et d'autant plus dépendant.

Or ici la professionnelle ne semble pas contredire le discours de la personne âgée, l'on suppose donc qu'elle a elle-même pu constater cette dégradation, celle-ci entraîne une prise en charge plus conséquente pour les professionnels qui s'occuperont de ce résident d'où l'intérêt de transmettre ce type de discours aux pairs.

Enfin, circonstance malheureusement incontournable d'un établissement hébergeant des personnes âgées en fin de vie, le professionnel considère également pertinent de partager les discours concernant des remerciements qu'il a eus avec la famille du résident en cas de décès :

(12) Professionnelle 7 : Alors rien de particulier à Tambour ${ }^{5}$ on a nettoyé la chambre de madame a tout qui est à désinfecter on l'a désinfecté dans la salle de bain on attend que ça sèche pour ranger et verticalisateur

Professionnelle 1 : Ouais il faut le récupérer

Professionnelle 7 : Sinon y a rien

Professionnelle 1 : Très bien

Professionnel non identifié : Je savais pas moi

Professionnelle 1: C'est écrit dans les transmissions

Professionnel non identifié : Compliqué

Professionnel non identifié : En plus j'ai vu un mail dans ce sens

\footnotetext{
${ }^{5}$ Les secteurs de l'EHPAD portent le nom d'instruments de musique.
} 
Professionnel non identifié : Monsieur L demande de nuit si on peut lui donner avant

Professionnelle 1 : Nan nan mais y avait j'l'avais j'l'ai noté donc sa famille remercie le personnel

Les discours des familles des résidents peuvent également être transmis à la communauté comme c'est ici le cas pour des remerciements après un décès. L'on peut s'imaginer également que tous les discours en liaison avec la famille et qui impactent les soins et la prise en charge de la personne âgée seront également transmis lors des transmissions orales.

Le dialogisme interdiscursif a donc une réelle fonction au sein des transmissions. Il permet de tenir au courant l'ensemble des professionnels des discours antérieurs qui sont importants pour le soin et l'aide de la personne âgée que ce soit au sujet de la santé du résident, d'un changement de comportement, d'un refus, de douleur exprimée par le résident ou de tout autre discours que le professionnel jugera opportun de transmettre aux autres professionnels.

Son utilisation est non seulement requise mais indispensable au bon fonctionnement d'une équipe qui se consacre au soin et à l'aide des personnes âgées. En effet, pour que le passage des informations soit fluide, la transmission des discours antérieurs est un incontournable de ce type de réunion professionnelle. Ces transmissions sont aussi le lieu de prise de décision collective. En effet, c'est aussi lors des transmissions orales que les professionnels débattent et prennent des décisions collectives sur la base notamment des discours qui ont été transmis :

(13) Professionnelle 2 : Madame D euh c'est euh... Martine qui a fait sa toilette ce matin et elle dit que elle avait eu des selles beaucoup de selle et elle dit que dans ses selles elle a trouvé euh quelque chose qui ressemblerait à un glaire mais de sang, avec du sang

Professionnelle 1: D'accord

Professionnelle 2 : Voilà donc euh

Professionnelle 1 : Bah me faire un test avec l'eau oxygénée

Professionnelle 2: Ah ah! J'avais raison

Professionnel non identifié : J'te l'ai dit

Professionnelle 2: Tu peux expliquer à Martine que si on mettait de l'eau oxygéné ça moussait et ça voyait qu'y avait la présence de sang

Professionnelle 1: De sang tout à fait et dans ces cas-là on peut faire des examens euh complémentaires

Professionnelle 2: Ok on en a ici de l'eau oxygénée?

Professionnelle 1 : Ouais on en a à l'infirmerie

Ici une professionnelle transmet le discours qu'elle a eu avec une collègue au sujet de l'inquiétante apparition de ce qui s'apparente à des glaires de sang dans les selles d'une résidente. En transmettant ce discours au sein des transmissions orales, le professionnel soumet au jugement et à l'approbation des autres professionnels les mesures à prendre dans ce genre de situation. Il sera ainsi décidé collectivement de mettre de l'eau oxygénée sur les glaires de sang pour s'assurer qu'il s'agit effectivement de sang, si la solution mousse alors le professionnel constatera la présence de sang et d'autres mesures seront probablement prises ultérieurement collectivement.

\subsection{Dialogisme interlocutif}

Dans le corpus récolté au sein du milieu professionnel, nous ne trouvons presque aucune trace de dialogisme interlocutif qui est nous le rappelons, un discours anticipé avec une réponse de l'allocutaire elle aussi anticipée. Cela semble cohérent, nous sommes ici dans une logique d'action et de discours réel, les discours anticipés ou imaginés n'ont pas une grande place au sein de l'action en contexte réel comme cela peut être le cas en formation. 


\subsection{Dialogisme intralocutif}

Le dialogisme intralocutif fait référence aux dits intérieurs, aux discours internes notamment dans la justification des actions et des processus mentaux associés au sein de discussions entre professionnels. Nous en retrouvons ainsi de façon prévisible uniquement au sein des transmissions orales pour ce qui concerne le milieu professionnel.

Il pourra s'agir de la justification de la gestion d'un résident non coopératif pour faire sa toilette :

(14) Professionnelle non identifiée: Donc Monsieur T sa toilette elle n'est pas encore faite parce il est très agressif ce matin et quand je suis arrivée en fait il essayait de se mettre dans son fauteuil dans sa chambre et j'lui ai dit bah attendez Henry on va se rafraîchir un p'tit peu euh et euh il écoutait rien de ce que je lui disais donc bah j'l'ai aidé à le mettre dans son fauteuil et puis il le ramènera

La professionnelle se justifie car elle n'a pas pu réaliser la toilette du résident et elle verbalise donc le discours intérieur qui l'a menée à cette conclusion auprès des autres professionnelles « il écoutait rien de ce que je lui disais donc bah j'l'ai aidé à le mettre dans son fauteuil et puis il le ramènera ». Cette justification se révèle indispensable, autrement il pourrait s'agir d'un oubli et donc d'une erreur professionnelle lors de la prise en charge.

Dans cet autre extrait, la professionnelle verbalise son discours intérieur au sujet des circonstances qui l'amènent à réaliser un change (en observant la ligne ou indicateur de saturation) :

(15) Professionnelle 1: Bah c'est ce que je disais en fonction du degré de saturation si vous voyez que elle est pas elle est que le degré de fin la ligne de saturation du change elle est correcte c'est tout vous laissez dans ces caslà sa p'tite toilette elle sera faite au coucher avec le change

Le langage parlé n'utilise pas souvent la forme réfléchie (« ce que je disais » à la place de « ce que je me disais ») c'est du moins ce que nous avons pu constater parmi les nombreux phénomènes d'« ellipse », de raccourcis induits par les transmissions orales. Ainsi même si la professionnelle commence sa prise de parole avec « ce que je disais » dans les faits, c'est la première fois qu'elle verbalise cette réflexion à ses collègues, elle est donc issue de son dit intérieur. Lorsqu'une réflexion est verbalisée lors des transmissions orales c'est qu'elle n'a pas été partagée au groupe précédemment. En effet les informations données en transmissions orales sont inédites sinon la prise de parole de la professionnelle n'aurait tout simplement pas lieu d'être. La verbalisation du discours intérieur permet ainsi à la professionnelle d'expliquer la logique derrière ses gestes afin que les autres puissent les reproduire.

Il peut également s'agir d'interrogations que le professionnel souhaite verbaliser aux autres professionnels :

(16) Professionnel non identifié : On se dit bah du coup on va mettre un bleu si on repasse une heure après ou alors on met le mauve tout de suite et on vérifie mais au final du coup tu le rechanges à $16 \mathrm{~h}$ et tu le rechanges au coucher parce qu'il a de nouveau fait, est-ce c'est pas une perte de change du coup? Est-ce que ce serait pas mieux plutôt que $19 \mathrm{~h}$ se dire bah $21 \mathrm{~h} 22 \mathrm{~h}$ au premier à la première ronde de nuit?

Ici il s'agit de s'interroger sur l'heure du change le soir aux autres professionnels, dans un raisonnement partagé issu du dit intérieur. Le « on » reflète la logique de réflexion collective pour les soins donnés à la personne âgée, les transmissions orales sont un lieu et un moment pour obtenir un consensus et une logique partagée. Les soins se coordonnent ainsi à travers une réflexion collective et d'un groupe qui partagent la même culture professionnelle.

Il peut aussi s'agir de conclusion verbalisée par le professionnel comme c'est le cas ici : 
(17) Professionnelle 8: Monsieur J a donné son (liquide) ${ }^{6}$ j'l'ai laissé agir un p'tit peu et puis ça a rien donné donc du coup j'lui ai pas donné ses cachets parce que c'était au moment de la toilette donc j'ai dit ça sert à rien si c'est pour euh toute la journée, voilà

S'agissant de langage parlé, le verbe dire, bien qu'il ne soit pas utilisé à la forme réfléchie (je me suis dit) introduit le discours intérieur de la professionnelle ( $c f$. langage parlé et la forme réfléchie). Dans le nord de la France où le corpus a été récolté, il existe également une confusion fréquente entre le verbe avoir et le verbe être, nous avons pu ainsi voir dans le corpus de nombreuses erreurs de cet ordre. Il est ainsi plus qu'invraisemblable que la professionnelle ait exprimé son incapacité en présence du résident comme ici mentionnée "ça sert à rien si c'est pour euh toute la journée ». Lors des transmissions orales, la professionnelle justifie ainsi ses actes en présentant son dit intérieur, ici le fait de ne pas avoir donné de médicaments au résident.

De même, il peut s'agir de conclusions collectives ou individuelles que le professionnel tire de son raisonnement aux autres professionnels :

(18) Professionnel non identifié : Après j'dis maintenant qu'il a un si il devient incontinent fécal on est plus obligé de le prendre en charge à 7 h20

On trouvera également au sein des transmissions orales des dits intérieurs sous la forme de conclusions générales et de prise de décision verbalisées qui impliquent d'autres professionnels :

(19) Professionnelle 1: Donc voilà parce qu'après, on, me, ouais pour la nuit mais du coup ça veut dire que l'équipe de toilette elle fera p'us de toilette j'dis bah nan ça change pas grand-chose dans le sens où dans tous les cas Monsieur $S$ il est y a une soignante qui est sollicitée pour sa toilette donc il reste encore deux autres soignants pour faire d'autres toilettes de personnes qui sont déjà réveillées

Professionnelle 1 est l'infirmière coordinatrice de l'EHPAD, elle soumet donc son raisonnement personnel aux autres membres du personnel dans une logique de décision partagée et consensuelle, ici la toilette pour un résident ne sera plus effectuée à priori par l'équipe de nuit. La référence au « dit intérieur » ou dialogisme intralocutif est donc un élément fondamental dans une profession avec une logique de consensus et de partage des connaissances. Cette verbalisation du « dit intérieur » permet aux autres professionnels de partager des raisonnements qui les conduisent par exemple à la non-réalisation d'un soin et à la justification de la pratique professionnelle mais aussi à la verbalisation de processus mentaux et des actions associées, dans une optique de bonne pratique, de reproduction et d'homogénéisation.

\section{Conclusion}

$\mathrm{Au}$ sein de la pratique professionnelle, l'utilisation du dialogisme interdiscursif est indispensable à la bonne pratique, d'abord pour transmettre les discours de la famille du résident aux professionnels. Mais c'est surtout au sein des transmissions orales qu'il est repéré et abondamment utilisé, que ce soit pour mentionner un discours avec une personne âgée qui s'est montrée agressive, le discours d'une personne âgée qui exprime des douleurs, le discours d'un résident qui souhaite un changement dans ses soins, de tous les changements issus de discours antérieurs que le professionnel considère pertinents de partager aux autres

\footnotetext{
${ }^{6}$ Le mot en parenthèses n'est pas audible, nous ne pouvons donc pas savoir ce qui a justifié le fait de ne pas donner des médicaments aux résidents, peut-être la mise en place d'un autre dispositif médical remplaçant les médicaments à administrer?
} 
professionnels. C'est au cours de ces transmissions que le professionnel transmet les communications qu'il a eues avec le résident mais également celles qu'il a eues avec d'autres professionnels ou les informations qu'il a reçues et qu'il considère pertinent de partager aux autres professionnels.

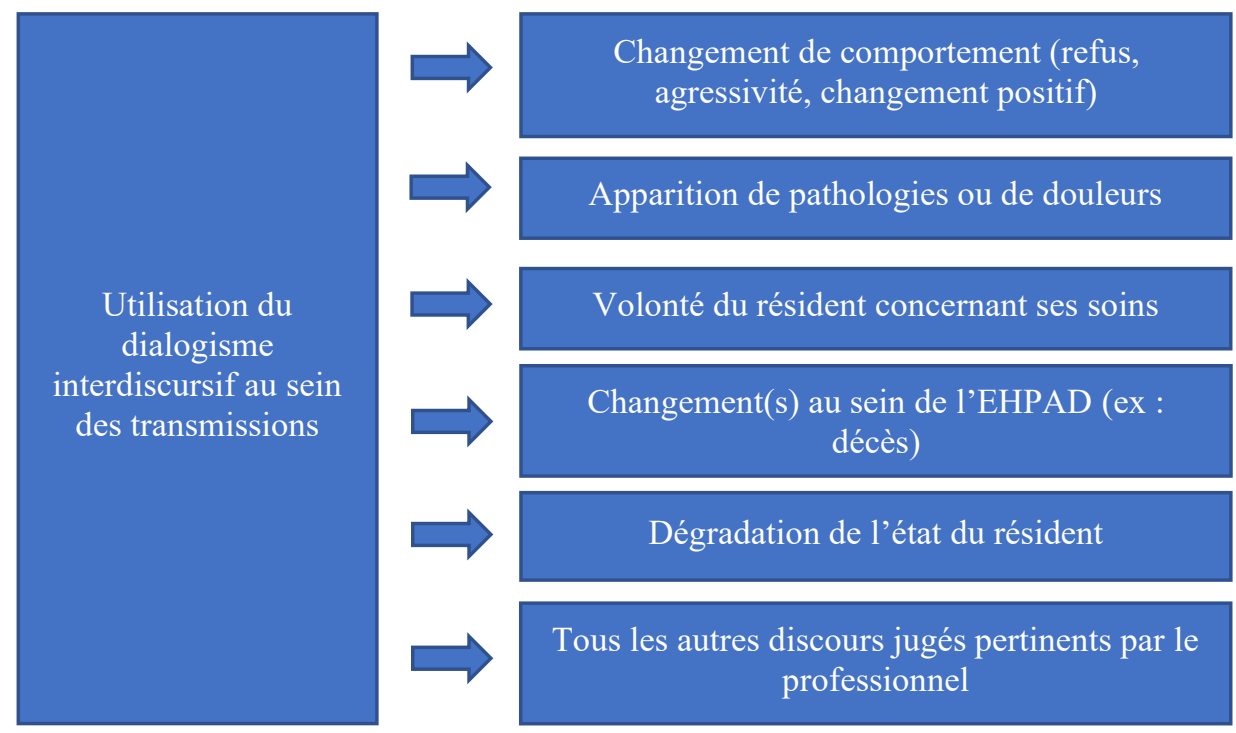

Figure 3. Utilisation du dialogisme interdiscursif au sein des transmissions.

Par ailleurs, comme nous le constatons dans cette figure résumant l'utilisation du dialogisme interdiscursif au sein des transmissions, les informations transmises ne le sont jamais au hasard. Rappelons que nous sommes dans une logique de soin et d'aide et qu'une personne extérieure à la pratique professionnelle serait bien incapable de transmettre les bonnes informations, les bons discours. Cette transmission répond toujours à une logique, à un raisonnement professionnel avec des codes implicites spécifiques à la pratique. C'est par exemple, transmettre les remerciements de la famille sans jamais mentionner directement le décès de la personne ou encore évoquer la volonté d'une résidente concernant ses soins et qui demande une chaise percée. Les transmissions orales sont métaphoriquement ce lieu de carrefour où s'échangent toutes les informations et discours indispensables aux bons soins du résident et à la bonne pratique. C'est ainsi notamment grâce au recours du dialogisme interdiscursif que se prennent des décisions collectives et consensuelles au sein des transmissions orales. Par ailleurs, c'est aussi au cours de ces transmissions que se partagent les raisonnements professionnels, ici nous sommes dans une forme de transmission avec le dialogisme intralocutif. Les transmissions orales sont un lieu où les professionnels mutualisent leurs raisonnements professionnels.

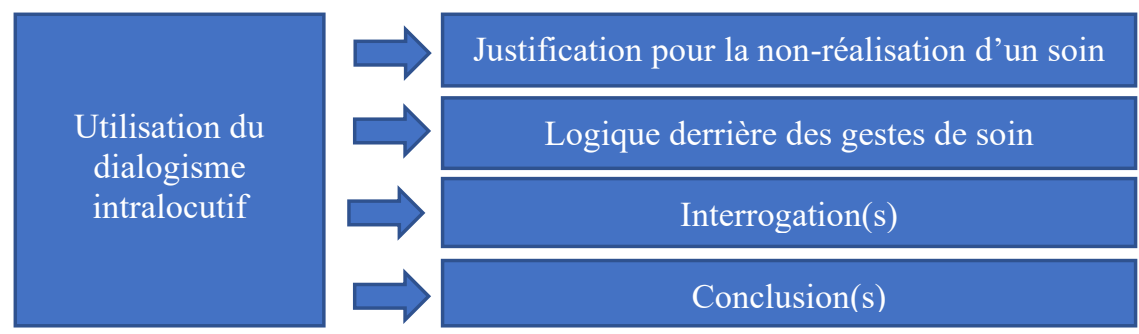

Figure 4. Utilisation du dialogisme intralocutif au sein des transmissions. 
Là encore il ne s'agit pas de transmettre tous les raisonnements pensés au sein de la pratique professionnelle (ce serait par ailleurs sans doute impossible) mais de communiquer ceux qui s'avèrent être pertinents comme la justification pour la non-réalisation d'un soin car la personne âgée s'est montrée non-coopérative ce jour-là par exemple ou encore d'expliquer le raisonnement, la logique derrière des gestes de soins.

L'utilisation des discours lors des transmissions orales répond donc à des implicites spécifiques à la pratique, les professionnels du soin et de l'aide comme ceux de la santé ne transmettent pas tous les discours perçus ou pensés à leurs pairs. Dans une logique de rentabilité et de productivité seuls certains discours sont transmis opportunément lors des transmissions orales, les discours choisis sont ceux qui permettront à terme, une meilleure prise en charge de la personne âgée dépendante. Nous avons pu également observer que les discours choisis par les professionnels ne concernaient pas uniquement l'état de santé de la personne âgée mais englobaient des problématiques qui allaient bien au-delà. L'étude du dialogisme et la problématique de l'interdiscusivité concernant les transmissions orales nous auront donc permis de comprendre que les communications interprofessionnelles répondent à des logiques implicites mais maitrisées par tous les membres de la communauté de pratique. Cependant et même si la compréhension des implicites de la pratique est intéressante notamment dans le cadre de formation (en particulier pour le recrutement de personnel étranger qui aurait plus de mal à les comprendre et les intégrer), il demeure parfois compliqué de repérer le dialogisme dans les discours professionnels telles que les transmissions orales. Comme nous avons pu le signifier notamment à cause des phénomènes induits par une prise de parole orale (phénomènes de réduction, d'ellipse, de modification en contexte parlé, etc.), il demeure difficile de saisir ce qui, dans le langage parlé, relève de discours notamment du dit intérieur. Déduire tous les implicites qui contrôlent l'utilisation des discours se relève ainsi très complexe. De plus comme toute culture, la culture professionnelle liée à la prise en charge des personnes âgées évolue, les impératifs, les implicites qui contrôlent la pratique peuvent se modifier sur un court laps de temps, c'est notamment ce que nous rappelle les évènements soudains comme la pandémie de COVID-19.

\section{Références}

Boutet Josiane. (1995). Paroles au travail. Paris : L'Harmattan.

Boutet Josiane, Gardin Bernard. (2005). Une linguistique au travail. In A. Borzeix, B. Fraenkel, Langage et travail Communication, cognition, action. Paris : CNRS Éditions. p. 89-111.

Bres Jacques. (2017). Dialogisme, éléments pour l'analyse. In N. Auger, S. Dufour, C. Parpette, Recherches en didactique des langues et des cultures. Paris : Les Cahiers de l'Acedle.

Charaudeau Patrick, Maingueneau Dominique. (2002). Dictionnaire d'Analyse du Discours. Paris : Seuil.

Grosjean Michèle, Lacoste Michèle. (1999). Communication et intelligence collective le travail à l'hôpital. Paris : Presses Universitaires de France.

Direction de la recherche, des études, de l'évaluation et des statistiques. (2016). Les conditions de travail en EHPAD vécues comme difficiles par des personnels très engagés. https://drees.solidarites-sante.gouv.fr/IMG/pdf/dd05.pdf, consulté le 29/04/2020

Lefelle Marie. (2019). Données authentiques dans le domaine de l'Aide à la personne et genre professionnel. In J. -J. Richer, A. Kaaboub. Action Didactique $n^{\circ} 3$, p. 126-144. http://univ-bejaia.dz/action-didactique/pdf/ad3/Lefelle.pdf 
Mourlhon-Dallies Florence. (2011). Entre aide et soin : spécificités du français langue professionnelle pour les employés au domicile de particuliers. In F. Haeuw, M. Ferrari, Répondre aux besoins linguistiques des salariés du particulier employeur: bilan d'une recherche action. Paris : Les éditions de l'institut FEPEM de l'emploi familial.

Sapir Edward. (2001). Le langage Introduction à l'étude de la parole. Paris : Petite bibliothèque Payot.

Trivalle Christophe. (2016). Gérontologie préventive - Éléments de prévention du vieillissement pathologique. Issy-les-Moulineaux : Elsevier Masson.

Zarifian Philippe. (1996). Essai sociologique sur le travail dans la grande entreprise industrielle. Travail et communication. Paris : PUF. 